\title{
Emerging Energy Management Standards and Technologies - Challenges and Application Prospects
}

\author{
Rumen Kyusakov, Jens Eliasson, \\ Jan van Deventer and Jerker Delsing \\ Luleå University of Technology \\ SE-971 87 Luleå Sweden \\ rumen.kyusakov@ltu.se
}

\author{
Robert Cragie \\ Gridmerge Ltd. \\ 89 Greenfield Crescent Grange Moor \\ Wakefield WF4 4WA United Kingdom \\ robert.cragie@gridmerge.com
}

\begin{abstract}
The continuously rising costs and the environmental impact of energy generation, transmission and consumption are a major concern for governments, industry and society alike. Among research in renewable energy sources as well as in energy efficiency of buildings, electrical appliances, vehicles etc., a considerable amount of attention has been devoted to effective energy management.

In this paper, we present a survey on emerging energy management standards with focus on enabling application layer Information and Communications Technologies (ICT) that are a central part of these standards. The presented work includes an analysis on the challenges, future trends, security and application prospects of energy management standards. As part of the survey, the emerging Open Automated Demand Response (OpenADR) version 2.0 and Smart Energy Profile (SEP) version 2.0 were identified as the most promising and complete solutions. The presented survey provides an important insight on the future developments in the area of energy management protocols and highlights a number of key ICT solutions and challenges.
\end{abstract}

\section{Introduction}

Enabling automatic exchange of information between different interdependent systems can optimize wide range of industrial processes. The access to dynamic pricing information and automatic load control can for example be used to employ methods that manage the electricity consumption or delivery in a way that lowers the costs and environmental impact of the consumed electrical energy [1]. The use of such two-way communication infrastructure for electrical power grids is called Smart Grid. The goal of the Smart Grid is to enhance reliability of electricity distribution, reduce peak demand, shift usage to offpeak hours, lower total energy consumption and carbon dioxide footprint.

The deployment of Smart Grid infrastructure encom- passes the coordination of the processes in a large number of systems: electricity producers (power plants and Distributed Energy Resources (DER)), electricity transmission and distribution, residential/commercial buildings and other energy consumers [2]. These systems have interdependencies with other systems that can be used to further increase the process optimization. For example, connecting district heating and cooling management systems with Smart Grid infrastructure can be used to reduce the peak energy demands especially when combined heat and power (CHP) plants are used [3].

Even small improvements in reducing the peak demands and energy footprint in residential and commercial buildings have substantial impact on the energy consumption. For example, the buildings sector in the U.S. accounted for almost $40 \%$ of all primary energy consumption in 2008 - that is $43 \%$ more than the transportation sector and $24 \%$ more than the industrial sector [4].

There are a number of problems in today's energy management systems. First, there is a discrepancy between the customer load and the value of energy at a given point in time. Unlike other consumer markets, the energy market is very inflexible in a sense that the demand is not sensitive to supply constraints and prices which is caused by poor communication. It is not unusual that the energy demand is at its highest during peak prices periods. Second, the increase in the number of DER, including generation (e.g. wind and solar power, industrial co-generation) as well as storage (e.g. electrical vehicles), makes the coordination of supply and demand to maintain the reliability of the distribution network challenging.

It is clear that a communication infrastructure, that connects all the heterogeneous systems and facilitates an automatic coordination, monitoring and control based on predefined energy management policies, is needed in order to address the problems of the energy market. However, the currently available communication standards for energy management does not encompass all the aspects and requirements of such communication infrastructure. Instead, they are defined within a narrow scope e.g. transmission (IEC 61970), distribution (MultiSpeak, IEC 
61968), substation automation (IEC 61850), Advanced Metering Infrastructure (AMI) (M-Bus, IEC 62056, Universal Metering Interface (UMI)) or supervisory control and data acquisition (SCADA) for power system automation (IEC 60870-5, IEC 60870-6, DNP3/IEEE 18152010).

While the interoperability between these different systems and the large number of devices is crucial, the communication technologies in use are often incompatible, based on proprietary solutions, unable to support heterogeneous PHY/MAC layers (e.g. SEP v1.0) or impossible to scale to all use cases. One parameter connected to that is the efficiency of communication protocols i.e. low bandwidth, CPU and memory requirements, which are needed to support the growing number of devices that are taking part in the energy management systems.

\subsection{Internet of Things in energy management}

The concept of distributed energy management requires a number of systems that traditionally have been isolated to cooperate and exchange information. The sources and scope of the information flows are very heterogeneous and include measurements and sensor data from various sparsely distributed processes. In gathering the data and controlling these processes, the Internet of Things (IoT) technology can be a viable and cost-effective solution [5]. A key enabling technology for low-cost IoT energy management deployments is the use of wireless communication for sensor and control data. In this case, the overall cost of deployment is much lower as compared to wired installations.

The use of IoT technology also imposes some additional constraints, such as low bandwidth communication, limited radio coverage, resource-constrained devices etc. This might prohibit, or limit, the use of standardized security protocols, full-fledged communication stacks, and require new, more efficient data communication solutions.

\subsection{Problem formulation}

The main objective of this work is to investigate the state of the art and the newly emerged application layer technologies used for energy management and the Smart Grid in particular. Broader discussions on the available demand-side management techniques along with motivation, benefits, opportunities and challenges of their application are presented by Strbac [6]. The analysis presented in his study highlights the lack of ICT infrastructure as a major challenge for demand-side management.

Nevertheless, there are no systematic studies on the requirements and possibilities of using available and emerging ICT in energy management applications. The analysis in [7] investigates the communication requirements and challenges from very high level and abstract perspective without connecting the results of the study to concrete protocols and technologies. In contrast, the work presented in [8] is limited to ANSI C12.22 and Session Initiation Protocol (SIP) and does not consider the latest developments in data representation formats and application layer protocols.

For that reason, the work presented here is dedicated to ICT challenges and enabling technologies in some of the recent energy management standards. The considered research questions include:

- What are the ICT and security challenges when applying the emerging energy management standards?

- How can recent developments in efficient data formats and application protocols be used to mitigate these challenges?

- What is the level of support for IoT devices by the investigated standards and technologies?

\section{Requirements}

This paper considers a set of technical requirements used as a standpoint during the analysis of the energy management standards. The requirements are focused on the ICT and do not include general functionality that the standards should cover as presented in [7]. A mapping between an exhaustive list of key energy management functions, similar to the one presented in [9], and the concrete standards is very important research topic. However, this calls for defining representative set of use cases, market assessments etc. and it is out of the scope of this work.

The set of requirements that we consider builds on the work presented in [8] and includes the following:

- Interoperability - it is vital that the ICT technologies support end-to-end communication services in a wide range of application domains for the energy management such as Smart Grid, district heating/cooling, ventilation, building and industrial automation. The interoperability requires the use of standard-based and open communication technologies that support heterogeneous communication links.

- Efficiency - low overhead in terms of network bandwidth, CPU, RAM and programming memory is essential for the IoT devices.

- Scalability - the technologies must scale to large networks - both in terms of geographical distribution and number of nodes.

- Security

- Efficient installation and network management

\section{Communication technologies}

Building the whole Smart Grid communication infrastructure from scratch would require huge R\&D and development efforts. Therefore, it is almost universally agreed 
that the Internet, including network protocols and related infrastructure, should be used as communication backbone for the energy management systems.

Our primary interest is on high level standards and technologies that define data serialization techniques and exchange patterns rather than frame and packet definitions on PHY/MAC, network and transport layers. Nevertheless, this section describes various low-level communication technologies that are important for the energy management systems. The support for these networking technologies by the application layer energy management standards is a key criterion for comparison and analysis used later on in the paper.

Another motive for the broader investigation in this section is security - the application layer energy management standards depend entirely on the lower layers network protocols for securing the communication. Traditionally, building automation systems and sensor networks have been closed networks running on specific wiring and therefore have never really required many of the security controls that are taken for granted in the Internet. However, with the advent of wireless technology and widespread access to the Internet, it has become increasingly important to design security into the communication protocols from the start.

\subsection{Physical and data link layers}

Wired technologies such as Ethernet and powerline provide high robustness and bandwidth with relatively low latency. Offices, residential buildings and industrial sites often have Ethernet installations. Powerline communication is also gaining acceptance in the networking community, especially since 6LoWPAN was selected in the new G3-PLC standard to transmit IP data over a standard powerline installation.

Being primarily of academic interest, wireless solutions have rapidly gained acceptance. The IEEE 802.11 family of standards (WLAN) and low power radio communications based on IEEE 802.15.4 such as WirelessHART, ISA100.11a and ZigBee are now widely adopted for industrial and consumer use. WiFi, with bandwidths of up to several hundreds Mbit/s, can support virtually any energy management service with low real-time requirements. On the other hand, the bandwidth of IEEE 802.15.4-based radio solutions, as well as some other lowpower radio technologies such as $\mathrm{Z}$-Wave, is constrained to no more than $250 \mathrm{kbit} / \mathrm{s}$. Battery powered sensors and actuators are usually equipped with these low power radios and employ duty cycling and sleeping schedules to limit the periods in which the radio is turned on, hence reducing the power consumption. The combination of low bandwidth and battery power limitations requires networking, security and application layer protocols that are very light-weight in terms of message sizes and computational resources.

Many wireless MAC/PHYs specify a network access mechanism to ensure that only trusted devices join the network. The Extensible Authentication Protocol is often used to carry handshaking, which can check credentials of joining devices and provide a key which can be subsequently used to provide link-layer security. An example of this is Wireless Protected Access (WPA) in the 802.11 specification and Protocol for Authentication and Network Access (PANA) [10].

A higher level of security at the link layer is also common. For example, 802.15.4-2006 specifies the use of AES-CCM, which is a NIST-approved mode of operation [11] using the AES-128 block cipher [12]. Similarly, 802.11 specifies the use of AES-CCM amongst other protocols. AES-CCM uses a symmetric key and provides confidentiality, integrity and data origin authentication. When used with a unique frame counter, it also provides replay protection. Many of the chipsets which support these wireless protocols have the AES-CCM and AES128 security engines built into the hardware, thus simplifying the implementation of a secure link layer protocol.

\subsubsection{Network layer}

IP protocol is the de facto network layer solution on the Internet. It has gained wide acceptance in industrial applications and for highly constrained networked devices where the interoperability with external systems is essential. The adoption of the latest version of the protocol (IPv6) has also progressed as it is crucial for supporting the huge number of IoT devices. Adaptation layer technologies such as IETF 6LoWPAN and lightweight routing protocols such as IETF RPL (both adopted in ZigBee IP) allow low-power devices with limited processing capabilities to take part in the Internet of Things.

IP-based networks can use IPsec, which is a suite of IETF specifications that provides confidentiality, integrity and data origin authentication to IP datagrams through security associations. The security association is established using a handshake based on one of a number of cipher suites, which passes credentials either one way or both ways. The credentials are checked and then used to establish one or more keys, which form the basis of the security association.

\subsubsection{Transport layer}

TCP and UDP are established transport layer protocols used in wide range of applications. Transport Layer Security (TLS) for TCP connections and Datagram Transport Layer Security (DTLS) for UDP serve as additional layers on top of the transport layer and provide confidentiality and integrity checking based on a secure session. The secure session is established in a similar way to the secure association for IPsec, i.e. using a handshake based on one of a number of cipher suites. 


\subsubsection{Presentation layer and cryptography}

Two of the functions of the presentation layer are machine independent data representation and encryption/decryption. A key requirement for the data representation is interoperability of the exchanged information. As a result, distributed information systems often use XML, JSON or similar serialization of the data. The use of open serialization formats is a central part of the web service application layer technology where the messages are often stateless and exchanged over HTTP protocol with URL used for resource addressing. The main drawback of the web service protocols is the size of the messages which makes them a challenging approach for battery powered embedded devices. Nevertheless, recent developments in embedded web service protocols has shown promising results in this area [13].

Encryption and decryption functions use cryptography to provide confidentiality of the data. Public and symmetric key cryptography are also used as a basis for the authentication and key establishment protocols described earlier. Public key cryptography relies on difficult to solve mathematical relationships such as integer factorization and the discrete logarithm problem. However, these are often based on very large prime numbers, which require a considerable amount of processing power and produce large key sizes. Elliptic Curve Cryptography is a public key cryptography which requires much smaller key sizes and much less processing power and is therefore more appropriate for the often constrained nature of the IoT devices.

Symmetric key cryptography generally requires much smaller key sizes and processing power than public key cryptography. The main issue is distributing the same confidential information to all parties involved in the communication.

\section{Data models}

For complete interoperability between different energy management standards and systems supporting demandside management, the common representation of the exchanged information - both syntactical and semantical, is of utmost importance. Various data serialization formats are in use today such as ASN.1, data tables (ANSI $\mathrm{C} 12.19$ ) and different proprietary solutions. Another wellestablished and gaining wider acceptance technology for defining the representation of the communicated data is XML and XML Schema definition language. One important benefit of this approach is the possibility to structure the data with human-readable meta-data in the form of hierarchies of elements and attributes. The use of informative names of the elements and a structure that represents the properties of the real-world objects of interest makes the development work, connected to wiring different application interfaces, much easier.

An important aspect of the data modeling is semantic definitions, i.e. a common understanding of the mean- ing of exchanged information. The semantic definitions are often represented with a natural language, UML models and ontology databases intended for system developers. While the human readable descriptions are important, they often contain ambiguities and are impossible to process by machines. One approach to address these problems is the use of ontologies that augment the syntactical description of the data with references to well-known ontology databases. As the amount of data generated by all devices and systems part of the Smart Grid infrastructure will be tremendous, the use of well-defined ontologies for the data representation should also be considered. This could bring more flexibility to the system operations and allow for automated reasoning when processing the information.

\section{Energy management standards}

A key requirement for successful application of demand-side energy management is interoperability of the communication infrastructure. Interoperability depends upon common standards [14], which define how data is exchanged, what data formats are used and how different systems react to certain events according to a common energy management policy that assures reliability of the energy management system.

This section presents the investigated standards and technologies. The focus is on the aspects that were defined in the problem formulation section: the use of ICT, application scope and support for constrained networked devices (IoT).

\subsection{IEEE Smart Grid interoperability guide}

This standard contains guidelines and best practices for interoperability of Smart Grid infrastructure [15]. It only serves as a high level architectural view of the system components and is not sufficient per se for deriving concrete implementations nor ensuring interoperability. However, it provides engineering principles and design evaluation criteria for the other concrete standards included in this study.

\subsection{OpenADR version 2.0}

Open Automated Demand Response (OpenADR) standard version 2.0 is an evolution and extension to the first version of the specification developed by Demand Response Research Center at Lawrence Berkeley National Laboratory. OpenADR 2.0 is supported by OpenADR industry alliance and developed as part of the OASIS Energy Interoperation 1.0 standard published in February 2012 [16].

The core of the OpenADR 2.0 standard is a set of data models and exchange patterns that define standard demand-response (DR) signals and the interfaces between energy markets (dynamic and transaction pricing information), Independent System Operators (ISO), utilities, 
Distributed Energy Resources (DER) and energy consumers (industrial/residential buildings). The data models (Energy Market Information Exchange (EMIX) and WSCalendar) are defined using XML Schema definition language and hence all the DR information is communicated as XML messages. OpenADR 2.0 does not specify the communication medium and technologies used below the application layer. While the standard is designed to be agnostic regarding the actual transport scheme and communication protocols, the exchange patterns (interfaces) are based on Service Oriented Architecture (SOA) and are defined using Web Service Description Language (WSDL).

OpenADR 2.0 specifies different levels of conformance that together with the OpenADR certification program guarantees interoperability between different OpenADR 2.0 products. In order to claim full compliance however, the implementations should be compatible with WS-I Basic Profile, which requires the use of SOAP web services. Deploying SOAP web services on resourceconstrained devices is challenging [17] and therefore the OpenADR scope does not cover IoT applications. While mainly targeted at Smart Grid deployments, OpenADR 2.0 can be applied to other distribution networks such as natural gas and water.

\subsection{SEP version 2.0}

Smart Energy Profile (SEP) version 2.0 [18] is an application layer specification for devices that are part of Demand Response and Load Management (DRLC) programs. It is supported by the Consortium for SEP 2 Interoperability (CSEP) and has been identified by the National Institute of Standards and Technology (NIST) as a primary candidate specification for energy information and control on the consumer side.

The specification includes smart metering, pricing and DRLC applications for devices in residential and light commercial buildings operating on a Home Area Network (HAN), sometimes called Premises Area Network. In this way, the scope of SEP2 differs from that of OpenADR: SEP2 is focused on HAN devices while OpenADR is designed for high level communications between the AMI Systems, utilities' communication networks and ISOs.

SEP2 builds on top of the IP protocol and is agnostic of the underlying PHY/MAC layers. Therefore, it supports Ethernet, WiFi, powerline communications and different low-power radio technologies. For the data exchange, SEP2 relies on RESTful web services that are defined as a set of CRUD operations (create, read, update, and delete) on a remote resource identified by a URL. The implementation of RESTful web services can use different application protocol bindings with HTTP being the most widely adopted where the CRUD operations are mapped to the HTTP methods GET, PUT, POST, and DELETE. As with OpenADR, the data model in SEP2 is defined using XML Schema definition language and maps directly to the IEC 61968 standard. As SEP2 is targeted at IoT deployments, the need for efficient representation of the XML messages is important, therefore the SEP 2 specification has identified Efficient XML Interchange (EXI) [19] as the most promising solution for XML compression.

\subsection{Building and industrial automation protocols}

The success of demand side energy management depends on the active participation of energy consumers in controlling their loads in response to changes in energy prices. For the building sector, this requires a high level of automation of load control in which human interactions are restricted to choosing comfort and efficiency levels from an intuitive graphical user interface. In the case of industrial sites, the dynamic energy price can be included as a variable in the process control algorithms. Therefore, there is a need for close integration between the protocols delivering demand/response signals and the systems interpreting and reacting on these signals. The following paragraphs summarize the ICT used in some widely adopted automation protocols:

BACnet can use a number of PHY/MAC layers including Ethernet, RS-232, LonTalk etc. and work is being done to support ZigBee Building Automation profile as well. Some of the latest developments of BACnet are directed towards more open communication technologies such as BACnet over TCP/IP networks (BACnet/IP) and even defining web service profile (BACNet/WS).

LonWorks consists of a communication protocol (with associated transport channels) known as LonTalk (standards ISO/IEC 14908-1 to -4) and a proprietary application layer protocol. There are some initiatives to support tunneling of LonTalk over IP as well as providing web service interface to LonWorks.

ZigBee Home/Building Automation are two application profiles built on the ZigBee stack, which is in turn based on the IEEE 802.15.4 low-power wireless standard. They do not support IP and standard web services without protocol translation on a gateway device.

oBIX is an OASIS specification that aims at providing an open, XML- and web service-based exchange of information for building control systems.

IEC 61499 used with a widely adopted compliance profile (CPFD) requires the use of IP, TCP/UDP and a proprietary data encoding based on modified ASN.1

MTConnect is based on web service technology with HTTP RESTful interface and data serialization based on XML and XML Schema.

OPC UA defines several profiles that run on top of TCP/IP and use either XML or proprietary encoding format, as well as either HTTP-SOAP web services or proprietary transport scheme. 


\section{Technology analysis}

Successful deployment of Smart Grid infrastructure requires interoperability of the communication systems that are part of it - starting from high-end enterprise management systems running on powerful servers or even cloud infrastructure to embedded sensors and actuators. Interoperability, on the other hand, depends on common standards, certification and conformance test programs. Looking at the scope of SEP2 and OpenADR2 for example (Figure 1), the end-to-end interoperability must include a gateway device that translates the OpenADR2 demandresponse signals to SEP2 load control and pricing messages. This requires certification and conformance test programs for end-to-end interoperability that go beyond the scope of a single standard. As such, the certification activities of the OpenADR alliance and CSEP must be aligned in the future to meet this challenge.

NIST's Smart Grid Interoperability Panel (SGIP) highlighted a number of gaps and inconsistencies along with action plans for the available communication, data representation, smart metering and energy management standards. SGIP also identified the need for open ICT that are proven to scale well over huge networks. Example of such proven technologies are the communication protocols that underpin the Internet - TCP/IP protocol stack, $\mathrm{XML}$ on the presentation layer and web services on the application layer. The trend towards the use of Internet protocols can be seen in the latest energy management standards (SEP2 and OpenADR2) and to some extend in the building and industrial automation sector. However, the use of open communication standards, XML and web services (SOAP- or RESTFul-based) in particular, brings new challenges that would make the transition from proprietary ICT problematic. The first group of challenges is related to the resource requirements of such solutions and the second one is related to security.

\subsection{ICT resource requirements}

The switch from proprietary solutions to a standard web service technology will often require more powerful hardware and hence increase the per unit cost of the final products. For example, a smart meter equipped to support SOAP-based OpenADR2 signals or SEP2 RESTful web services needs much more RAM, computing power and battery power compared to a meter that supports EN 13757-3 (the application layer protocol of M-Bus). Even more challenging is the use of web services over lowpower and low-bandwidth radio links that are often used for remote meter readings [20]. In many cases, protocol gateways will be required to translate between low-level specialized protocols and SOA-based energy management communications. This will inevitably complicate the installations and make the maintenance of the system troublesome.

Nevertheless, some newly emerged ICT can be used to increase the efficiency of the web service implemen-

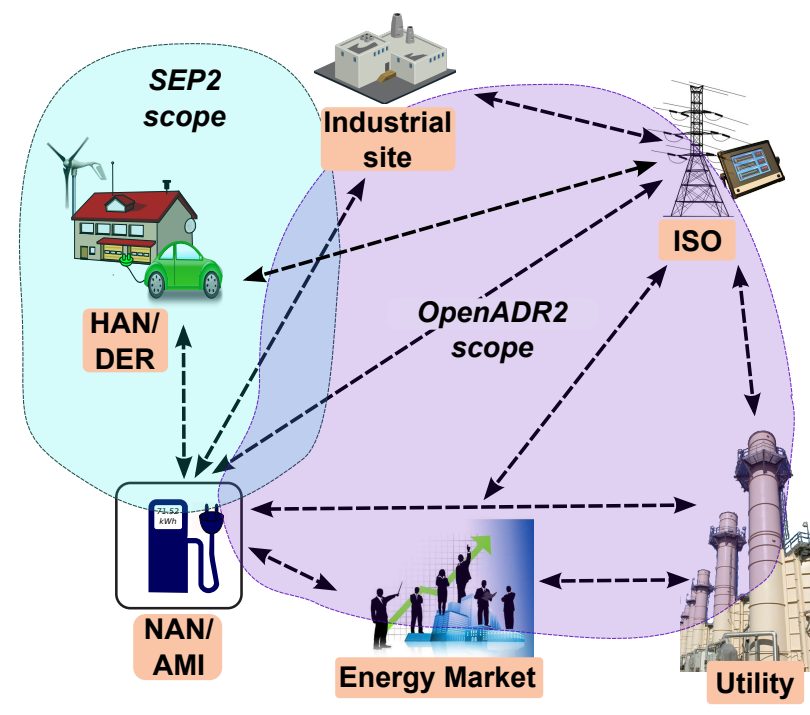

\section{Figure 1. Application scope of OpenADR2 and SEP2}

tations. Application protocol, called Constrained Application Protocol (CoAP), is currently under development by IETF and is specially designed for IoT deployments. It is a binary REST protocol that resembles HTTP but provides some features for embedded networked devices that go beyond the capabilities of HTTP e.g. multicast and event-driven asynchronous message passing. The advantages of CoAP over HTTP for IoT, including quantitative measurements of the gained efficiency, are presented by Colitti et al. [21].

Another important technology for embedded web services is efficient representation of the XML. A primary candidate for that is Efficient XML Interchange (EXI) specification - W3C recommendation since 2011. The studies performed by W3C EXI working group showed compression of up to 100 times as well as multifold processing speed increase compared to XML (depending on the encoding options and the document structure) [22, 23]. By using EXI, the energy management protocols will ensure interoperability with enterprise management systems while keeping the overhead of IoT deployments to a minimum.

For quantifying the EXI compression for a concrete protocol, a sample of 9 SEP2 messages typical for a smart thermostat were selected and converted to an EXI representation using the latest SEP2 XML schema definition.

As shown in Table 1, on average the EXI messages are only $7.89 \%$ of their XML counterparts with an average size of 40 bytes. By using EXI serialization, the SEP2 messages are very likely to fit into a single 6LoWPAN packet which is an important requirement for battery powered sensor nodes.

Another IoT resource requirement is the programming memory of the EXI implementations. The programming 
Table 1. SEP2 EXI/XML sample messages

\begin{tabular}{|l|r|r|}
\hline Encoding type & Avg. comp. [\%] & Avg. size [bytes] \\
\hline \hline XML (text) & 100.0 & 462 \\
\hline EXI (schema) & 7.89 & 40 \\
\hline
\end{tabular}

memory is proportional to the complexity and size of the XML schema as its definitions are stored in the form of programming language constructs. We performed tests with two embedded EXI implementations available as open source: EXIP ${ }^{1}$ and WS4D-uEXI ${ }^{2}$. The measurements of the programming memory size were done with a sample EXI decoder for SEP2 messages on a 32 bit Linux PC. The programming memory for the EXIP sample application was measured $2702 \mathrm{~KB}$ while for WS4D-uEXI the size was $392 \mathrm{~KB}$. These results reveal the difference in the way the XML schema definitions were stored: EXIP uses regular grammars in the form of $\mathrm{C}$ structures while WS4D-uEXI uses finite state machines implemented with hierarchy of switch statements. In both cases there are methods that can be used to lower the size of the programming memory such as storing only the XML schema definitions that are used by the device, representing multiple read-only definitions with a single structure etc.

Introducing new protocols and data serialization formats will bring a new challenge to the Smart Grid implementations - namely the migration of legacy deployments in utilities and ISO management systems. On the other hand, the investments in this migration could be partly covered by the increased reliability and lower maintenance cost of the future Smart Grid.

\subsection{Security}

Keeping the Smart Grid secure has to do with many different aspects that cover the communication security but also the practical application of security policies by all the actors in the system [24]. In this section, we examine some of the key security challenges for the emerging energy management standards.

\subsubsection{Ease of installation vs security}

One of the biggest challenges facing devices in Smart Grid infrastructure, sensor networks and appliances is the need for proven security often with a very limited or nonexistent user interface. In the past, default passwords or codes have been used (e.g. the "0000" pin used in Bluetooth pairing) but these have severe limitations.

Most computing devices today have a high resolution graphical display and a keyboard or touchscreen and therefore entering a credential such as a username and password is easy. Moreover, it is often the user of the computing device who is being authenticated and subsequently authorized for, e.g. a banking transaction, and not

\footnotetext{
${ }^{1}$ EXIP - http://exip.sourceforge.net/

${ }^{2}$ WS4D-uEXI - http://code.google.com/p/ws4d-uexi/
}

the device itself.

Devices running in a HAN or Smart Grid network may need themselves to be authenticated and subsequently authorized. An example is an electric vehicle; it may be eligible for cheap electricity but it is important to know that the device which is plugged in and drawing a large current is in fact an electric vehicle and not for example a large battery which can then be used to power a home instead of taking power from the grid.

Public key cryptography can be used to install a device certificate in the device, which is signed in a Public Key Infrastructure (PKI). The device certificate identifier is given out-of-band to the utility which then sends it to the metering device. When the vehicle is plugged in, it sets up a secure session based on its device certificate and the metering device can then be sure the device is genuine. No username or password is needed in this case. This technique can be applied to all HAN devices that need validating in themselves, as opposed to user validation.

Other techniques for devices without any user interface exist. Near-field communications (NFC) is a rapidlygrowing wireless technology which can transfer information wirelessly over a short distance. This makes it difficult to eavesdrop and therefore it can be used to configure devices securely with a master unit by simply holding them in close proximity. Similar approaches for registering security credentials are for example RFID tags, barcodes or QR codes.

\subsubsection{Prominent security threats}

The Smart Grid provides a large and varied attack space for attempted security breaches. Threats may come from many sources. Given the wide distribution of devices into homes, the most likely attacker will be the consumers themselves, trying to obtain cheaper bills. Smart Grid devices form part of critical infrastructure, therefore they will be a likely target for cyber terrorists who wish to cause major disruption by e.g. taking out electricity supplies. Attacks include eavesdropping whereby an attacker can understand confidential information, manin-the-middle attacks whereby an attacker can manipulate data or masquerade as a legitimate device and injection attacks whereby an attacker can inject spurious data into the network to cause disruption.

The security protocols already mentioned should be used at different layers to prevent eavesdropping, man-inthe-middle and injection attacks. Firewalls and air gaps must exist in the networks to ensure that security domains are distinct and any traffic passing between the domains is very carefully policed. Access control must be in place to ensure only privileged users and devices have authorized access to data.

\section{Discussion}

A common trend among energy management protocols is the use of proven ICT for ensuring scalable and secure 
communication. By use of compression schemes such as 6LoWPAN, IPv6 is slowly getting wider acceptance on the network layer. The use of XML and XML Schema definition language for representing the data (e.g. smart meter readings, pricing information, load control) is another widely adopted approach. In order to achieve the resource efficiency required for IoT deployments, current efforts are focused on the use of compression schemes for the verbose XML messages. One promising solution for efficient representation and processing of XML data is the EXI binary format.

While having potential benefits, semantic descriptions based on machine processable ontology databases are not part of the energy management standards on the presentation layer. This might change in the future as the need for more automated and flexible energy management communication infrastructure becomes evident.

Web services is another technology that is underlying the emerging SEP2 and OpenADR2 standards. One promising technology for lowering the resource requirements on the application layer is the CoAP protocol that can be used to implement RESTful as well as SOAP-based web services.

The security aspects in energy management domain are very important side of all standardization efforts. The major challenge in this respect is the need for high level of security for devices with limited resources that have no user interface and are required to support cost-effective installation procedures. One way to lower the resource requirements for these devices is to use lighter cryptographic algorithms such as Elliptic Curve public-key cryptography and hardware security engines built into the chipsets. On the other hand, near-field communications and barcodes can be used to configure security credentials and simplify installations.

\section{Acknowledgment}

This work is partly funded by the European Commission and EU FP7 project IMC-AESOP (www.imcaesop.eu). We thankfully acknowledge the constructive feedback of the anonymous reviewers.

\section{References}

[1] M. Albadi and E. El-Saadany. A summary of demand response in electricity markets. Electric Power Systems Research, 78(11):1989 - 1996, 2008.

[2] H. Farhangi. The path of the smart grid. Power and Energy Magazine, IEEE, 8(1):18 -28, january-february 2010.

[3] R. Aringhieri and F. Malucelli. Optimal operations management and network planning of a district heating system with a combined heat and power plant. Annals of Operations Research, 120:173-199, 2003. 10.1023/A:1023334615090.

[4] Buildings Energy Data Book, 2010. U.S. Department of Energy.
[5] J. Liu, X. Li, X. Chen, Y. Zhen, and L. Zeng. Applications of internet of things on smart grid in china. In Advanced Communication Technology (ICACT), 2011 13th International Conference on, pages $13-17$, feb. 2011.

[6] G. Strbac. Demand side management: Benefits and challenges. Energy Policy, 36(12):4419-4426, 2008.

[7] F. Bouhafs, M. Mackay, and M. Merabti. Links to the future: Communication requirements and challenges in the smart grid. Power and Energy Magazine, IEEE, 10(1):24 -32, jan.-feb. 2012.

[8] J. Wang and V. Leung. A survey of technical requirements and consumer application standards for IP-based smart grid AMI network. In Information Networking (ICOIN), 2011 International Conference on, pages $114-119$, jan. 2011.

[9] J. Quittek, R. Winter, T. Dietz, B. Claise, and M. Chandramouli. Requirements for energy management, July 2012.

[10] D. Forsberg, Y. Ohba, B. Patil, H. Tschofenig, and A. Yegin. Protocol for Carrying Authentication for Network Access (PANA), 2008.

[11] M. Dworkin. NIST SP 800-38C: Recommendation for Block Cipher Modes of Operation: the CCM Mode for Authentication and Confidentiality. Technical report, NIST, 2007.

[12] NIST FIPS-197: Advanced Encryption Standard, 2001.

[13] Z. Shelby. Embedded web services. Wireless Communications, IEEE, 17(6):52 -57, 2010.

[14] R. DeBlasio and C. Tom. Standards for the smart grid. In Energy 2030 Conference, 2008. ENERGY 2008. IEEE, pages $1-7$, nov. 2008.

[15] IEEE Guide for Smart Grid Interoperability of Energy Technology and Information Technology Operation with the Electric Power System (EPS), End-Use Applications, and Loads, 102011.

[16] Energy interoperation version 1.0, Feb 2012.

[17] R. Kyusakov, J. Eliasson, J. Delsing, J. van Deventer, and J. Gustafsson. Integration of Wireless Sensor and Actuator Nodes with IT Infrastructure Using Service-Oriented Architecture. IEEE Transactions on Industrial Informatics, 2012.

[18] Smart energy 2.0, 2012.

[19] Efficient XML Interchange (EXI) Format 1.0, March 2011.

[20] C. Groba and S. Clarke. Web services on embedded systems - a performance study. In Pervasive Computing and Communications Workshops (PERCOM Workshops), 2010 8th IEEE International Conference on, pages 726 731, march 2010.

[21] W. Colitti, K. Steenhaut, N. De Caro, B. Buta, and V. Dobrota. Evaluation of constrained application protocol for wireless sensor networks. In Local Metropolitan Area Networks (LANMAN), 2011 18th IEEE Workshop on, pages 1 -6 , oct. 2011.

[22] G. White, J. Kangasharju, D. Brutzman, and S. Williams. Efficient XML Interchange Measurements Note. Technical report, W3C, 2007.

[23] C. Bournez. Efficient XML Interchange Evaluation. Technical report, W3C, April 2009.

[24] H. Khurana, M. Hadley, N. Lu, and D. Frincke. Smartgrid security issues. Security Privacy, IEEE, 8(1):81 -85, jan.-feb. 2010. 